\title{
Do we need dedicated cardiac SPECT systems?
}

\author{
Piotr Slomka ${ }^{a}$ \\ a Department of Medicine, Cedars-Sinai Medical Center, Los Angeles
}

Received Sep 11, 2019; accepted Sep 12, 2019

doi: 10.1007/s12350-019-01921-7

\section{See related article, pp. 1323-1330}

Dedicated cardiac SPECT scanners have significantly improved in recent years, ${ }^{1}$ reducing imaging time and radiation dose. In particular, cardiac scanners have entered clinical practice, ${ }^{2,3}$ which use solid-state Cadmium-Zinc-Telluride (CZT) crystal detectors and feature innovative gantry designs, focusing on the heart. These new systems allow improvement in photon sensitivity (5-8 times higher) ${ }^{4,5}$ for cardiac imaging, as well as image resolution (up to 2 times higher). ${ }^{5}$ While solidstate CZT detectors are more expensive than traditional $\mathrm{NaI}(\mathrm{Tl})$ crystal detectors with photomultipliers, they do have important imaging advantages. The CZT detectors have improved energy response, which significantly reduces the scatter component of measured data. They also offer superior intrinsic spatial resolution compared to conventional Anger cameras. The use of less-bulky detectors (no need for photomultipliers) has resulted in more compact scanner size which has facilitated smallfootprint dedicated cardiac camera designs with custom imaging geometries and high-sensitivity collimation. There is a growing evidence, including large multicenter studies, that these solid-state SPECT systems demonstrate excellent diagnostic and prognostic utility in cardiac imaging. ${ }^{6-8}$

In the last few years, the equipment companies have also introduced general-purpose CZT-based SPECT systems to enable applications other than cardiac imaging. These cameras benefit from improved energy and image resolution due to the CZT detectors and have demonstrated improved image quality in early clinical

Reprint requests: Piotr Slomka, Department of Medicine, Cedars-Sinai Medical Center, Los Angeles, USA; slomkap@cshs.org

J Nucl Cardiol 2021;28:1331-3.

1071-3581/\$34.00

Copyright (C) 2019 American Society of Nuclear Cardiology. implementation as compared to general-purpose Angerbased systems. ${ }^{9,10}$ GE has introduced general-purpose CZT-based cameras, the Discovery NM/CT 670 and 870, equipped with conventional collimators and CT attenuation correction. These cameras are not only designed for general-purpose imaging but also targeting cardiac imaging applications. The conventional collimator design of these cameras may avoid the difficulties in cardiac imaging, specific to the multipinhole collimation and small field-of-view on the dedicated cardiac cameras. ${ }^{11}$

It is important, however, to point out that the general-purpose CZT cameras offer the same photon sensitivity as the conventional Anger systems, because the count sensitivity is related to the collimator and not the crystal. Thus, dedicated CZT cameras with specialized collimators can achieve much higher count sensitivity, consequently allowing faster acquisitions and lower injected doses. In particular, the 19-pinhole collimator, with each pinhole operating at a different focal depth, allows stationary image acquisition. Photons are acquired simultaneously through all the pinholes, allowing improved sensitivity as compared to the conventional camera with parallel collimators and rotating heads. For example, the reported measured system sensitivity of the Discovery NM/CT 670 CZT camera was 78 counts/s/MBq ${ }^{12}$ compared to 460 counts/ $\mathrm{s} / \mathrm{MBq}^{5}$ for the dedicated GE $530 \mathrm{CZT}$ design. Thus, in absolute terms, the sensitivity of the general-purpose CZT scanner will be 5.9 times lower than that of the dedicated CZT scanner with pinhole collimation.

How does it translate to the routine day-to-day clinical cardiac imaging? In this issue of the Journal on Nuclear Cardiology, Gimelli et al. ${ }^{13}$ compare headto-head a CZT-based all-purpose SPECT camera (NM/ CT 670) and a dedicated CZT cardiac device (NM 530) for the myocardial perfusion and functional analysis. In a pilot sample of 19 patients, Gimelli et al. tested low 99mTc-tetrofosmin dose scans (148$185 \mathrm{MBq}$ during stress and 296-370 MBq at rest). The scans were obtained with same injection stress-first protocol on both cameras. They found excellent 
correlation for several key parameters (\% uptake at stress and rest, correlation in ejection fraction and myocardial mass) between the two CZT systems.

It is reassuring that the image quality and key quantitative parameters for cardiac SPECT images are similar on such diverse CZT-based designs. This is despite the use of different reconstruction methods and different filters and energy windows (10\% on NM/CT 670 vs $20 \%$ on NM 530) for these 2 scans. It should be also pointed out that the scan times on the generalpurpose CZT scanner (12.5 min and $10 \mathrm{~min}$ for stress and rest) were approximately $67 \%$ longer than on the dedicated CZT scanner ( $7 \mathrm{~min}$ and $6 \mathrm{~min}$ for stress and rest). In fact, the counts on the general-purpose scanner would be similar to counts obtained with 3.5 times lower dose on the dedicated 530c CZT scanner, considering an almost sixfold difference in sensitivity. It is encouraging to see that such low-count scans can be performed on the general-purpose scanner and result in a good agreement with the dedicated scanner.

So do we need dedicated cardiac CZT scanners for nuclear cardiology, if the general-purpose cameras can provide similar images? While the agreement found by Gimelli et al. in the pilot sample of patients is excellent, the dedicated scanners will allow lowering the dose more significantly (as low as $1 \mathrm{mCi}$ or $37 \mathrm{mBq}$ ) as shown in other studies ${ }^{14,15}$-or alternatively perform ultrafast scans (2 min scans). Further advances in reconstruction software should improve equally general-purpose and dedicated cardiac systems. Dedicated scanners will retain advantage of higher sensitivity for cardiac imaging due to optimized geometry and collimator, as it is not related to the use of the CZT crystal.

There are also other considerations. While the equipment cost was not discussed in the manuscript by Gimelli et al., it is likely that larger field-of-view CZT detectors may be more expensive then smaller cardiac CZT systems. Another important factor in deciding which SPECT scanner to use for cardiac patients may be the availability of the $\mathrm{CT}$ for attenuation correction and for additional cardiac CT testing, for improved diagnosis. The dedicated cardiac SPECT scanners were rarely equipped with CT, likely due to the cost. General-purpose SPECT cameras such as the one studied by Gimelli et al. often are configured with good-quality fast CT scanner-this has proven to be a popular option in oncological imaging. The combination of SPECT and CT imaging could allow quantitative SPECT imaging with CT used during reconstruction for advanced physical corrections (attenuation and scatter). It also could enable efficient hybrid imaging combined with diagnostic CT, for example calcium scan, or even utilizing CT attenuation scan for calcium scoring. ${ }^{16-18}$

\section{References}

1. Slomka PJ, Patton JA, Berman DS, Germano G. Advances in technical aspects of myocardial perfusion SPECT imaging. J Nucl Cardiol 2009;16:255-76.

2. DePuey EG. Advances in SPECT camera software and hardware: Currently available and new on the horizon. J Nucl Cardiol 2012;19:551-81.

3. Slomka PJ, Dey D, Duvall WL, Henzlova MJ, Berman DS, Germano G. Advances in nuclear cardiac instrumentation with a view towards reduced radiation exposure. Curr Cardiol Rep 2012;14:208-16.

4. Sharir T, Ben-Haim S, Merzon K, Prochorov V, Dickman D, BenHaim $S$, et al. High-speed myocardial perfusion imaging: Initial clinical comparison with conventional dual detector anger camera imaging. JACC Cardiovasc Imaging 2008;1:156-63.

5. Imbert L, Poussier S, Franken PR, Songy B, Verger A, Morel O, et al. Compared performance of high-sensitivity cameras dedicated to myocardial perfusion SPECT: A comprehensive analysis of phantom and human images. J Nucl Med 2012;53:1897-903.

6. Otaki Y, Betancur J, Sharir T, Hu LH, Gransar H, Liang JX, et al. 5-Year prognostic value of quantitative versus visual MPI in subtle perfusion defects: Results from REFINE SPECT. JACC Cardiovasc Imaging 2019. https://doi.org/10.1016/j.jcmg.2019.02.028.

7. Betancur J, Commandeur F, Motlagh M, Sharir T, Einstein AJ, Bokhari S, et al. Deep learning for prediction of obstructive disease from fast myocardial perfusion SPECT: A multicenter study. JACC Cardiovasc Imaging 2018;11:1654-63.

8. Betancur JA, Hu LH, Commandeur F, Sharir T, Einstein AJ, Fish $\mathrm{MB}$, et al. Deep learning analysis of upright-supine high-efficiency SPECT myocardial perfusion imaging for prediction of obstructive coronary artery disease: A multicenter study. J Nucl Med 2018;60:664-70.

9. Keidar Z, Raysberg I, Lugassi R, Frenkel A, Israel O. Novel cadmium zinc telluride based detector general purpose gamma camera: Initial evaluation and comparison with a standard camera. J Nucl Med 2016;57:259.

10. Goshen E, Beilin L, Stern E, Kenig T, Goldkorn R, Ben-Haim S. Feasibility study of a novel general purpose CZT-based digital SPECT camera: Initial clinical results. EJNMMI Phys 2018;5:6.

11. Fiechter M, Gebhard C, Fuchs TA, Ghadri JR, Stehli J, Kazakauskaite E, et al. Cadmium-zinc-telluride myocardial perfusion imaging in obese patients. J Nucl Med 2012;53:1401-6.

12. Takahashi M, Miyazaki Y, Kondo A, Ehara T, Koga K, Matsunari I. Performance evaluation of the discovery NM/CT 670 CZT. J Nucl Med 2018;59:1835.

13. Gimelli A, Liga R, Bertassi M, Kusch A, Marzullo P. Head-tohead comparison of a CZT-based all-purpose SPECT camera and a dedicated CZT cardiac device for myocardial perfusion and functional analysis. J Nucl Cardiol 2019. https://doi.org/10.1007/ s12350-019-01835-4.

14. Nakazato R, Berman DS, Hayes SW, Fish M, Padgett R, Xu Y, et al. Myocardial perfusion imaging with a solid-state camera: Simulation of a very low dose imaging protocol. J Nucl Med 2013;54:373-9.

15. Einstein AJ, Johnson LL, DeLuca AJ, Kontak AC, Groves DW, Stant J, et al. Radiation dose and prognosis of ultra-low-dose stress-first myocardial perfusion SPECT in patients with chest pain using a high-efficiency camera. J Nucl Med 2015;56:545-51.

16. Schepis T, Gaemperli O, Koepfli P, Rüegg C, Burger C, Leschka $\mathrm{S}$, et al. Use of coronary calcium score scans from stand-alone multislice computed tomography for attenuation correction of myocardial perfusion SPECT. Eur J Nucl Med Mol Imaging 2007;34:11-9. 
17. Isgum I, de Vos BD, Wolterink JM, Dey D, Berman DS, Rubeaux $\mathrm{M}$, et al. Automatic determination of cardiovascular risk by CT attenuation correction maps in Rb-82 PET/CT. J Nucl Cardiol 2017;25:2133-42.

18. Patchett ND, Pawar S, Miller EJ. Visual identification of coronary calcifications on attenuation correction $\mathrm{CT}$ improves diagnostic accuracy of SPECT/CT myocardial perfusion imaging. J Nucl Cardiol 2016;24:711-20.

Publisher's Note Springer Nature remains neutral with regard to jurisdictional claims in published maps and institutional affiliations. 